\title{
Anti-Neutrino imprint in Solar Neutrino Flare
}

\author{
D. Fargion* \\ Physics Department, Rome Univ.1 and INFN, Ple.A.Moro 2, 00185, Italy
}

(Dated: August 18, 2018)

\begin{abstract}
Future neutrino detector at Megaton mass might enlarge the neutrino telescope thresholds revealing cosmic supernova background and largest solar flares neutrino. Indeed the solar energetic $\left(E_{p}>100 \mathrm{MeVs}\right)$ flare particles (protons, $\left.\alpha\right)$ while scattering among themselves on Solar corona atmosphere must produce prompt charged pions, whose chain decays are source of a solar (electronmuon) neutrino "flare" (at tens or hundreds MeV energy). These brief (minutes) neutrino "burst" at largest flare peak may overcome by three to five order of magnitude the steady atmospheric neutrino noise on the Earth, possibly leading to their detection above detection thresholds (in a full mixed three flavor state). Moreover the birth of anti-neutrinos at a few tens MeVs is well loudly flaring above a null thermal "hep" anti-neutrino solar background and also above a tiny supernova relic and atmospheric noise. The largest prompt solar anti-neutrino "burst" may be well detected in future Super Kamikande (Gadolinium implemented) anti-neutrino $\overline{\nu_{e}}$ signatures mostly in inverse Beta decay $\left(\overline{\nu_{e}}+p \rightarrow n+e^{+}\right)$and rarely in higher energy muon, or even rarest tau neutrino leptons. Our estimate for the recent and exceptional October - November 2003 solar flares and January 20th 2005 exceptional flare might lead to a few events above or near unity for existing Super-Kamiokande and above unity for Megaton detectors. The $\nu$ spectra may reflect in a subtle way the neutrino flavor oscillations and mixing in flight. A comparison of the solar neutrino flare (at their birth place on Sun and after oscillation on the arrival on the Earth) with other neutrino foreground is estimated: it offers an independent track to disentangle the neutrino flavor puzzles and its most secret mixing angles. The sharpest noise-free anti-neutrino imprint maybe its first clean voice.
\end{abstract}

\section{NEUTRINO-ANTI-NEUTRINO SOLAR FLARE}

If during largest solar flare, of a few minutes duration, (1) the particle flux escaping the corona eruption and hitting later on the Earth, is 3-4 order of magnitude above the common atmospheric CR background (while

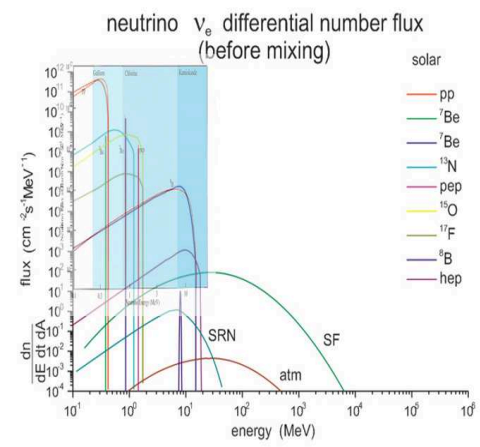

FIG. 1: The multi-component Neutrino flux due to Solar Nuclear Neutrino spectra and lines within the atmospheric (atm) Neutrino noises and Supernova Relic Spectra (SNR) (or DSNB, Diffuse Supernova Neutrino Background). The well known Bachall Solar Neutrino Spectra [3] overlaps an updated and wider neutrino spectra, containing the expected Solar Flare (SF) brightest fluxes [1]

*Electronic address: daniele.fargion@roma1.infn.it

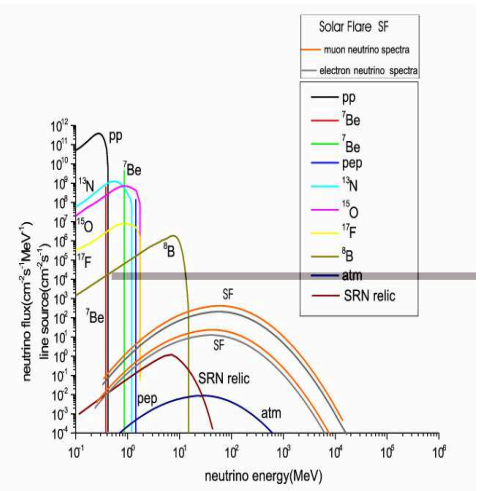

FIG. 2: As above, the $\nu_{e}$ signals of the Solar Flare $S F$ are shown after the flavor mixing for two extreme solar neutrino flare luminosity 11: just above or below the SK detection threshold.

showing similar spectra) and (2) if the flare particle interactions on the Sun corona is taking place as efficiently as in terrestrial atmosphere, than their secondaries by charged pions and muons decays, are leading to a neutrinos fluency on Earth comparable to one day terrestrial atmospheric neutrino activity [4]. One therefore may expect a prompt increase of neutrino signals of the order of one day integral events made by atmospheric neutrinos. In SK detector the signal is just on the edge, but as long as the author is aware, it has been never revealed. Sun density at the flare eruption might be diluted and pion production maybe somehow suppressed (by a factor $(0.1-0.05))$. This may be the reason for the null detection of huge event on Oct-Nov. 2003 and Jan. 2005. Unfortunately the neutrino signal at hundred $\mathrm{MeV}$ ener- 


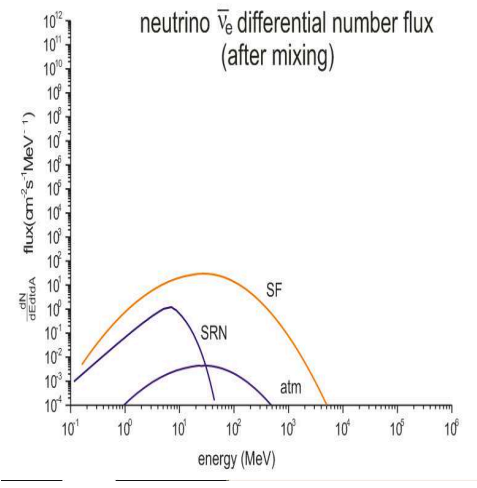

FIG. 3: As above SF $\overline{\nu_{e}}$ in noise-free spectra.

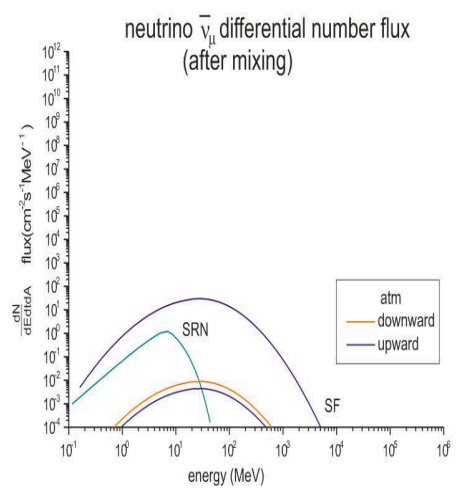

FIG. 4: As above for the $\overline{\nu_{\mu}}$ and $\overline{\nu_{\tau}}$ in noise-free background. Up-down atmospheric $\overline{\nu_{\mu}}$ asymmetry is also shown

gies is rare while the one at ten $\mathrm{MeV}$ or below is polluted by Solar Hep neutrinos or, in SK, by local neutrino reactor noise. Therefore Solar Flare expected signal (at softer spectra) maybe dominated by $30 \mathrm{MeV}$ neutrinos (made by non-relativistic pion decays) that might be better revealed by their anti-neutrino signature: it will be enhanced by Gadolinium presence in next SK detectors 11. Indeed Anti-neutrino electron imprint in Solar Flare may be well observable while searching for supernova relic anti-neutrino traces in the Universe 11]. The prompt SF signal may overcome the atmospheric noise just as the Tau Air-shower may overcome atmospheric muons [6].

There are two different SF (Solar Flare) neutrinos(see [1]): A brief and sharp solar flare, originated within the solar corona and a diluted and delayed terrestrial neu- trino flux, produced by flare particles hitting the Earth's atmosphere. We consider only the first. The main source of pion production is $p+p \rightarrow \Delta^{++} n \rightarrow p \pi^{+} n$; $p+p \rightarrow \Delta^{+} p \bar{\searrow}_{\searrow_{p+n+\pi^{+}}^{p+p+\pi^{0}}}$ at the center of mass of the resonance $\Delta$ (whose mass value is $m_{\Delta}=1232 \mathrm{MeV}$ ). As a first approximation the total pion $\pi^{+}$energy is equally distributed, in average, in all its final remnants: $\left(\bar{\nu}_{\mu}, e^{+}\right.$, $\left.\nu_{e}, \nu_{\mu}\right): E_{\nu_{\mu}} \geq E_{\bar{\nu}_{\mu}} \simeq E_{\nu_{e}} \simeq \frac{1}{4} E_{\pi^{+}}$. The flavor oscillation will lead to a decrease in the muon component and to a hardening of electron neutrino component spectra. While at the birth place the neutrino fluxes by positive charged pions $\pi^{+}$are $\Phi_{\nu_{e}}: \Phi_{\nu_{\mu}}: \Phi_{\nu_{\tau}}=1: 1: 0$, after the mixing assuming a number redistribution we expect $\Phi_{\nu_{e}}: \Phi_{\nu_{\mu}}: \Phi_{\nu_{\tau}}$ $=\left(\frac{2}{3}\right):\left(\frac{2}{3}\right):\left(\frac{2}{3}\right)$. On the other side for the antineutrino fluxes we expect at the birth place: $\Phi_{\overline{\nu_{e}}}: \Phi_{\overline{\nu_{\mu}}}: \Phi_{\overline{\nu_{\tau}}}$ $=0: 1: 0$ while at their arrival (within a similar flavor redistribution) $: \Phi_{\overline{\nu_{e}}}: \Phi_{\overline{\nu_{\mu}}}: \Phi_{\overline{\nu_{\tau}}}=\left(\frac{1}{3}\right):\left(\frac{1}{3}\right):\left(\frac{1}{3}\right)$. We considered the solar flare neutrino events due to these number fluxes following known $\nu$-nucleons cross-sections at these energies [7], 8], 10] at Super-Kamiokande II, finding a detectable signal: $N_{e v} \simeq 7.5 \cdot \eta\left(\frac{E_{F L}}{10^{31} \text { erg }}\right)$ (for more details and explanations see [1]). However the event expectation numbers at SK-II for solar neutrino burst assuming a more pessimistic detector thresholds calibrated with the observed Supernova 1987A event fluxes [1] is just at the detection edge compatible with null SK discover: $N_{e v \bar{\nu}_{e}} \simeq 0.63 \eta\left(\frac{\bar{E}_{\bar{\nu}_{e}}}{35 \mathrm{MeV}}\right)\left(\frac{E_{F L}}{10^{31} \mathrm{erg}}\right) ; \quad \bar{E}_{\bar{\nu}_{e}} \leq 100 \mathrm{MeV} ;$ $N_{e v \bar{\nu}_{e}} \simeq 1.58 \eta\left(\frac{E_{F L}}{10^{31} \text { erg }}\right) ; \bar{E}_{\bar{\nu}_{e}} \geq 100-1000 \mathrm{MeV} ;$ $N_{e v \bar{\nu}_{\mu}} \simeq 3.58 \eta\left(\frac{E_{F L}}{10^{31} \text { erg }}\right) ; \bar{E}_{\bar{\nu}_{\mu}} \geq 200-1000 \mathrm{MeV}$; where the efficiency factor $\eta \leq 1$. The background due to energetic atmospheric neutrinos at the Japanese detector is nearly 5.8 event a day corresponding to a rate $\Gamma \simeq 6.710^{-5} s^{-1}$ : the probability to find by chance one neutrino event within a $1-2$ minute $\Delta t \simeq 10^{2} s$ in that interval is $P \simeq \Gamma \cdot \Delta T \simeq 6.7 \cdot 10^{-3}$. For a Poisson distribution the probability to find $n=1,2,3,4,5$ events in a narrow time window might reach extremely small values: $P_{n} \cong e^{-P} \cdot \frac{P^{n}}{n !} \simeq \frac{P^{n}}{n !}=\left(6.7 \cdot 10^{-3}, 2.25 \cdot 10^{-5}, 5\right.$. $\left.10^{-8}, 8.3910^{-11}, 1.1 \cdot 10^{-13}\right)$. Therefore just a few events correlated to a Solar Flare will be a meaningful signal opening a window to novel Neutrino Astronomy.

This paper is dedicated to the memory of the flaring life of Jonathan Evron, fallen at brightest age 20 on $2 n d$ November 2005 for the Jewish State of Israel.
[1] D.Fargion,PoS, Hep (2005),336,1-8; JHEP06,(2004),045 ; D.Fargion,F.Moscato,Chin.J.Ast.Astrophys.3(2003)S75.

[2] M. Alessio, et. al Il Nuovo Cimento, 14C, 53-60, (1991).

[3] J.N.Bahcall et.al. Ap.J.555,990,2001

[4] T. K. Gaisser, T. Stanev PDG,PRD66,010001,182,2002

[5] W. C. Haxton, Phys. Rev. D, 36, 2283, (1987).

[6] D. Fargion et al.Ap.J.613,1285-1301;(2004)
[7] C. Bemporad,et. al. Rev. Mod. Phys. 74 297(2002)

[8] Strumia A., Vissani F., astro-ph/0302055 (2003)

[9] R. P. Lin, et all.Ap.J, 595, 2, Part 2;(2003).

[10] A.Bodek,et al. hep-ex/0309024 (2003)

[11] H. Yuksel,S.Ando, J.S.Beacom astro-ph/0509297 